\title{
study on the detection technology of DC penetration in coal mine
}

\author{
ShuangShuang Chen, Shucai Liu*, Yaoning Liu \\ School of Resource and Geosciences \\ China University of Mining and Technology \\ Xuzhou, China
}

\begin{abstract}
The paper deduced the electrical penetration potential equation of measuring point in point-source current field from the case study of conductive sphere, and proposed the question of the relationship between the response characteristics of electrical penetration curve and spatial location of anomalous body. Comparing the response characteristics of electrical penetration curves at different spatial position anomalies shows anomalous body at shallow electrical penetration curve showed opposite electrical characteristics, its abnormal response is strongest when close to the measuring point and is weakest near the middle of the working face. The results of 3-D resistivity inversion and numerical calculation show that the effective exploration depth of electrical penetration is $30 \%-40 \%$ of working face width, there exist exploration weakness in the middle of the working face.
\end{abstract}

Keywords-DC penetration; 3-D resistivity inversion; numerical simulation

\section{INTRODUCTION}

Mining DC penetration using data acquisition mode with arranging electrode in adjacent roadway of mining face, the mode can make full use of the mine confined spaces and provide a more comprehensive and accurate $3-\mathrm{D}$ geoelectric information compared traditional 2-D electrical prospecting vertical and horizontal (Liu et al.,2009), and the shielding property of current of the high resistance coal seam can be used to establish electric field directional (Liu et al.,1996; Han and Shi,200; Liu et al.,2001), its application prospects are good in detecting concealed structure on coal seam floor and mine water bursting and forecasting other geological hazards.

At present, mining DC penetration electrode arrangement and observation methods tends to approached perfection (Gyulai et al.,2013; Sasaki et al.,1993; Takacs,2012; Csokas et al.,1986), however, due to the lack of research on electric field distribution and the relationship between response characteristics of electrical penetration curve and spatial location of anomalous body, lead to the existing data processing and interpretation methods are single and lack of accuracy. The author deduce the electrical penetration potential equation of measuring point in point-source current field from the case study of conductive sphere, aiming at typical groundwater inrush models of fractured zone on working face floor, using 3-D finite element method for numerical simulation, analyzed and summarized the relationship be-tween response characteristics of electrical penetration curve and spatial location of anomalous body, and try to use 3-D resistivity inversion for electrical penetration data processing and interpretation, dis-cussed the feasibility of effective exploration depth of electrical penetration detection technology and the method of data processing and interpretation of the combination based on 3-D resistivity inversion and electrical penetration curve.

\section{Methodology}

As shown in the Fig. 1, the conductive sphere in half space model is constructed by utilized the shielding property of current of the high resistance coal seam, combined with the DC penetration parallel pole-dipole electrode arrangement. Assuming coal floor is homogeneous isotropic infinite medium, its resistivity is $\rho_{1}$, the conductive sphere on the floor which resistivity is $\rho_{2}$, radius is $r_{0}$, the $O^{\prime}$ as the origin( $O^{\prime}$ is the projection of $O$ on the working face), the direction of roadway and working face respectively as the $X$ axis and $Y$ axis coordinate system, the length of point source $A$ to $O$ is $d$, measurement point ( $M$ or $N$ ) from the center of the sphere is $r$, the length of $A$ to $M$ (or $N$ ) is $R$,the height of sphere top interface to working face is $h$ and the face width is $L$.

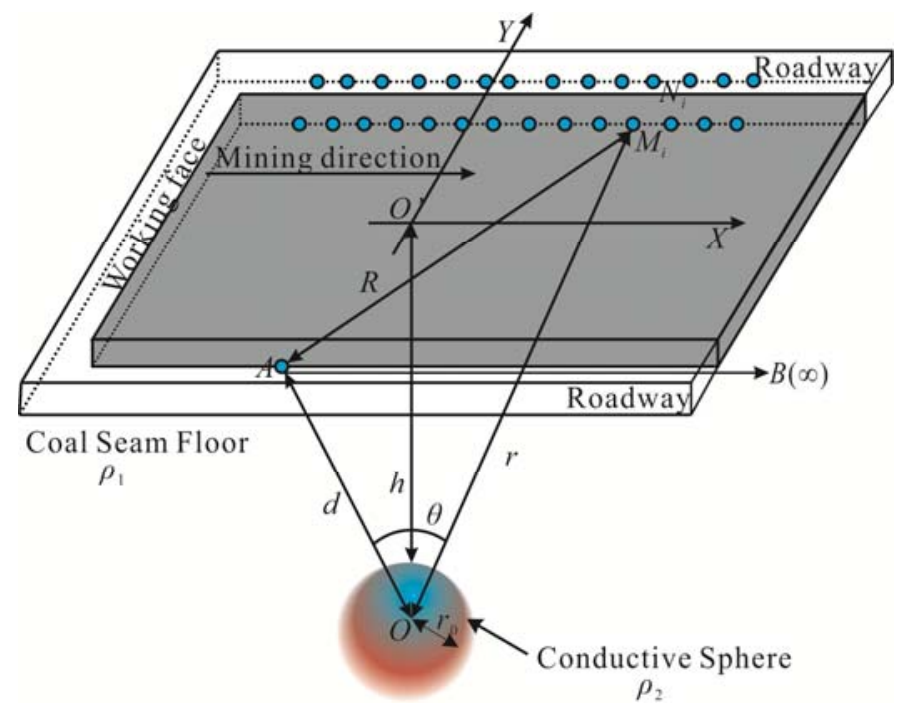

Fig.1 The model of conductive sphere in current field of point-source in half space 
According to basic equations of stable current field and boundary conditions can obtain the potential expression outside the sphere under half space, (1).

$$
U=\frac{I \rho_{1}}{2 \pi}\left[\frac{1}{R}+2 \sum_{n=0}^{\infty} \frac{n\left(\rho_{2}-\rho_{1}\right)}{\rho_{1} n+\rho_{2}(n+1)} \cdot \frac{r_{0}^{2 n+1}}{d^{n+1} r^{n+1}} \cdot P_{n}(\cos \theta)\right]
$$

Where, $P_{n}(\cos \theta)$ is n-order Legendre Polynomial of $\cos \theta$.

As shown in the Fig. 1, the horizontal distance between the origin $\left(O^{\prime}\right)$ and source electrode $(A)$ is $l$, measuring point along the $\mathrm{X}$-axis distance is $\mathrm{x}$, from the geometric relationship, can be obtained (2).

$$
\left\{\begin{array}{l}
R=\left(L^{2}+x^{2}\right)^{\frac{1}{2}} \\
r=\left[(L-l)^{2}+\left(h+r_{0}\right)^{2}+x^{2}\right]^{\frac{1}{2}} \\
d=\left[l^{2}+\left(h+r_{0}\right)^{2}\right]^{\frac{1}{2}} \\
\cos \theta=\frac{d^{2}+r^{2}-R^{2}}{2 d r}
\end{array}\right.
$$

According to (1) and (2), can be obtained the DC penetration potential approximate equation of measuring point, (3).

$$
\begin{aligned}
& U=\frac{I \rho_{1}}{2 \pi}\left[\frac{1}{\left(L^{2}+x^{2}\right)^{\frac{1}{2}}}+2 \sum_{n=0}^{\infty} \frac{n\left(\rho_{2}-\rho_{1}\right)}{\rho_{1} n+\rho_{2}(n+1)} \cdot P_{n}(\cos \theta) .\right. \\
&\left.\frac{r_{0}^{2 n+1}}{\left[l^{2}+\left(h+r_{0}\right)^{2}\right]^{\frac{n+1}{2}} \cdot\left[(L-l)^{2}+\left(h+r_{0}\right)^{2}+x^{2}\right]^{\frac{n+1}{2}}}\right]
\end{aligned}
$$

The measurement results of electric penetration can use two electrical parameters, rho and $\rho_{1}$ to express, (4).

$$
\left\{\begin{array}{l}
r h o=\frac{\Delta U_{\mathrm{MN}}}{I} \\
\rho_{s}=K \frac{\Delta U_{\mathrm{MN}}}{I}
\end{array}\right.
$$

Here, $\Delta U_{\mathrm{MN}}$ is electrode potential difference. Besides $I$ is current intensity and $K$ is array coefficient.

In the conductive sphere model, working face width is 100 $\mathrm{m} \quad(L=100 \mathrm{~m})$, and $r_{0}=30 \mathrm{~m}, M N=4 \mathrm{~m}, \quad l=50 \mathrm{~m}$. Moreover, $\rho_{1}=100 \mathrm{ohm}-\mathrm{m}, \rho_{2}=10 \mathrm{ohm}-\mathrm{m}$ (low resistance body) and $\rho_{3}=1000 \mathrm{ohm}-\mathrm{m}$ ( high resistance body). With the changing of depth: $h=0 \mathrm{~m}, h=10 \mathrm{~m}, \ldots, h=40 \mathrm{~m}, h=50 \mathrm{~m}$ respectively, the electrical penetration curves obtained by (3), as shown in Fig. 2. When conductive sphere in shallow, electrical penetration curves showed opposite electrical characteristics, and with the increasing of depth, the abnormal response of electrical penetration curves consistent with electrical property of conductive sphere.

The contour map of apparent resistivity rate of low resistance conductive spheres at the different spatial location of the working face floor, as shown in Fig. 3, ordinate is the distance between conductive sphere top surface and floor, abscissa is center point of conductive sphere, its value is apparent resistivity rate by measuring the electrode measured $(M N)$. When unilateral measuring, there has a strong low resistance abnormal response at outside of the source electrode $(A)$ and near the measuring electrode $(M N)$, and the abnormal response is gradually weakened with the increasing of depth, besides there has a strong high resistance abnormal response at working face range of about 10 80 $\mathrm{m}$ in shallow, however, with the increasing of depth high resistance response decreased and gradually transition to a low resistance response, but the low resistance response is relatively weak, as shown in Fig.3(a). When using bilateral measurement, apparent resistivity values present strong high resistance abnormal response at working face range of about $20 \sim 80 \mathrm{~m}$ in shallow, and there exist exploration weakness in the depths range of about 10 20 $\mathrm{m}$ at the middle of the working face, that is, in the area, the abnormal response are weak of low resistance and high resistance. If the apparent resistivity rate $10 \%$ (red and blue dashed line in Fig. 3) as to distinguish the abnormal line, hence, the effective exploration depth of DC penetration is about $30 \%-40 \%$ of working face width and increase at first then decrease from both sides toward the middle.
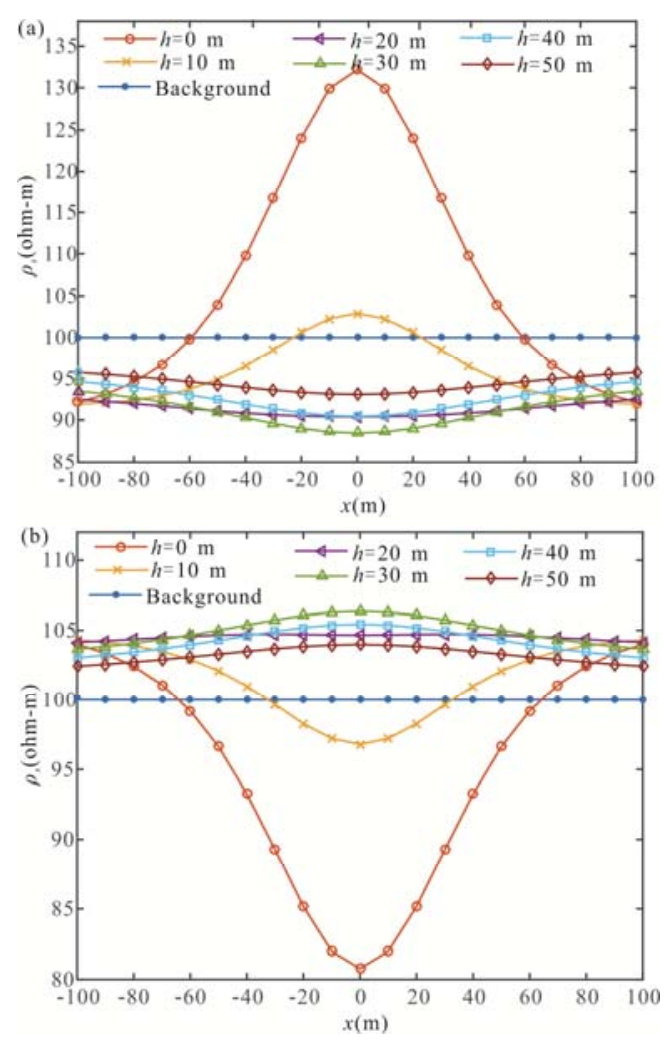

Fig. 2 The electrical penetration curves of conductive sphere at different depths: (a) Low resistance body; (b) High resistance body 


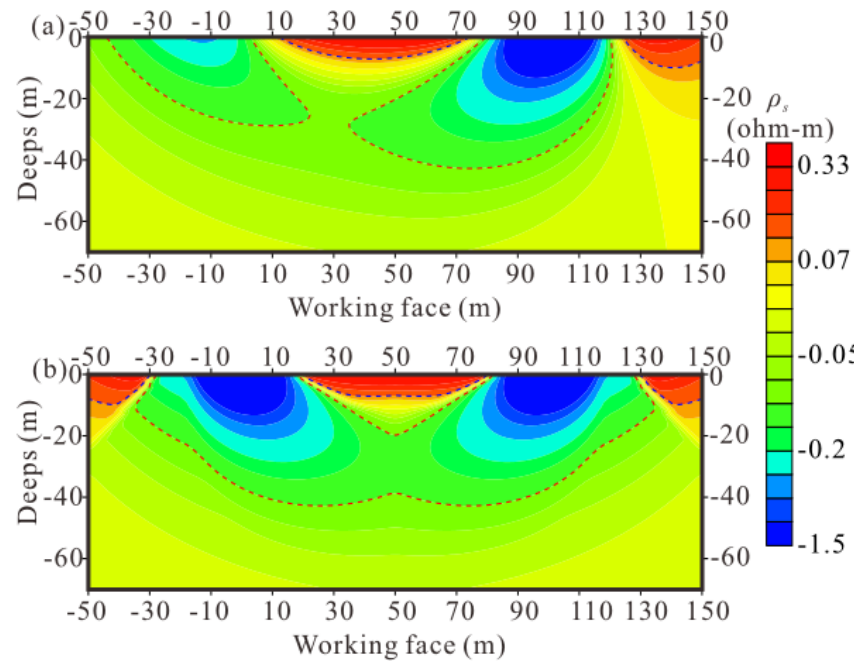

Fig. 3 The contour map of apparent resistivity rate of low resistance conductive spheres at the different spatial location: (a) Measuring at unilateral; (b) Measuring at bilateral

\section{MODELING}

The 3-D geoelectric model of fractured zone and electrode arrangement way, as shown in Fig. 4. The volume of the model and fractured zone are $400 \mathrm{~m} \times 300 \mathrm{~m} \times 200 \mathrm{~m}$ and 60 $\mathrm{m} \times 20 \mathrm{~m} \times 100 \mathrm{~m}$ respectively. Besides, the horizontal distance between the anomalous body and source electrode $(A)$ is $l$, and the vertical distance between top interface of anomalous body and coal floor is $h$, and other parameters consistent with the conductive sphere model.

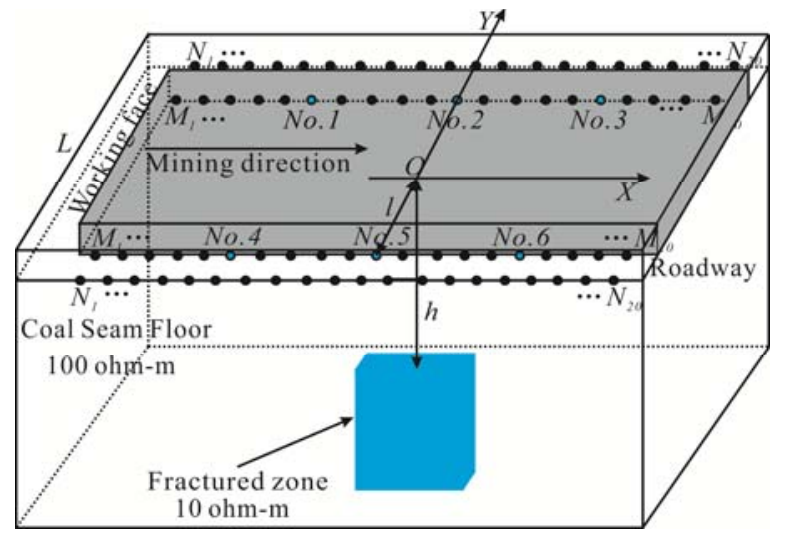

Fig. 4 Schematic diagram of 3-D geoelectric model and electrode arrangement

\section{A. Results of Forward Modeling}

According to electrode arrangement way in Fig. 4, No. 2 (or No. 5) as source electrode (A), and forward numerical simulation by 3-D finite element method. With the changing of spatial position of anomalous body, the apparent resistivity value are calculated and electrical penetration curves are drew in Fig. 5. When $l=50 \mathrm{~m}$, the electrical penetration curves of low resistance body at different depths all for low resistivity anomaly response, but in shallow, anomaly response is weak, and the abnormal saturation value of the apparent resistivity rate is about $16 \%$ when the depth is about $20 \mathrm{~m}$, as shown in
Fig. 5(a). According to the Fig. 5(b), when $h=20 \mathrm{~m}$, the electrical penetration curves all for low resistivity anomaly response. With the increasing of $l$, the apparent resistivity values of both sides increased and of the middle first decrease then increased. Anomaly response is strongest when the anomalous body near the measuring point $(l=90 \mathrm{~m})$ and is weakest near the middle $(l=40,50 \mathrm{~m})$ of the working face. Besides, the apparent resistivity rate is larger at the edge position of anomalous body (No. 8 and No. 14 measuring point), and it can divided the horizontal range of anomalous body.
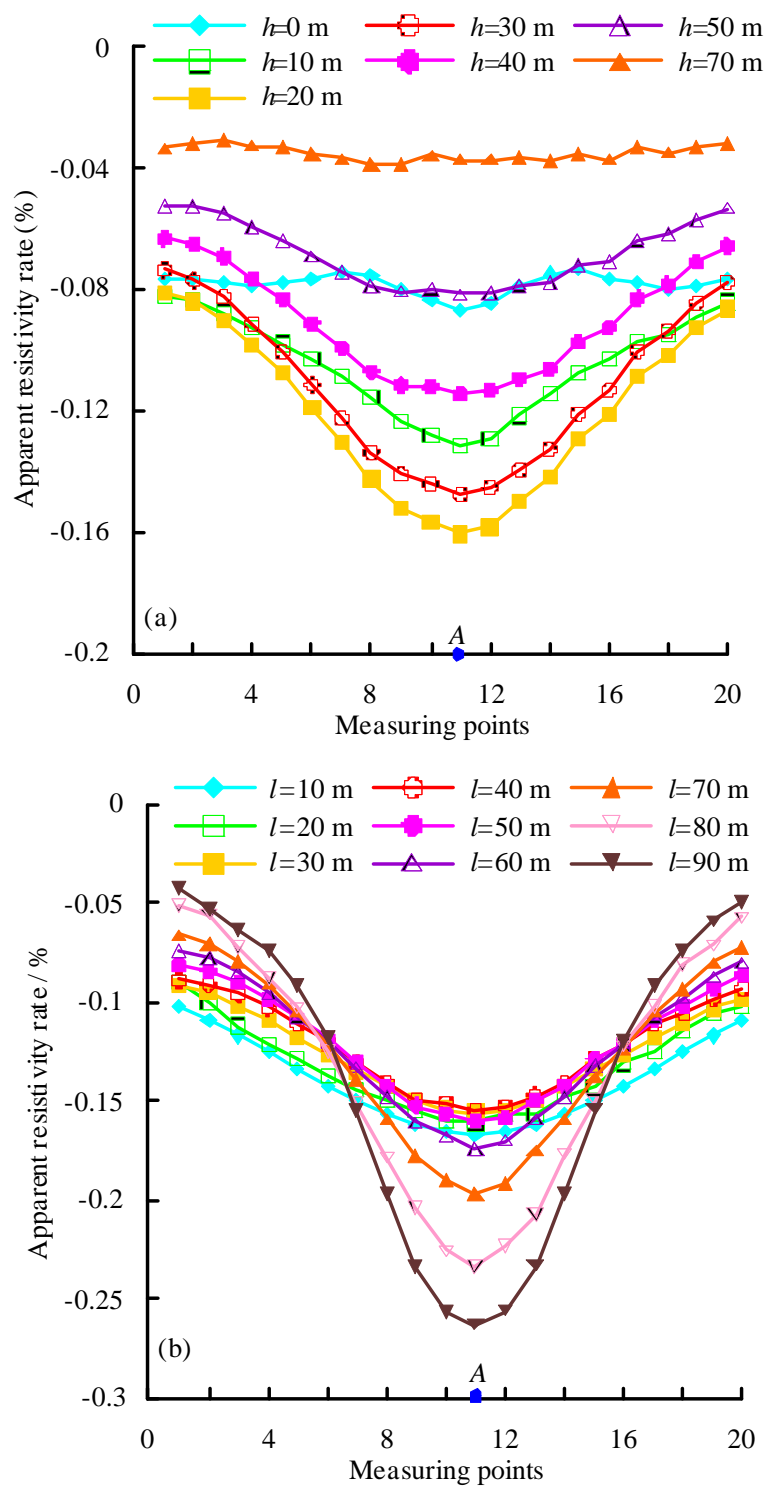

Fig. 5 Apparent resistivity rate curves of low resistance body at different spatial position: (a) $l=50 \mathrm{~m}$; (b) $h=20$

\section{B. Results of 3-D Resistivity Inversion}

According to electrode arrangement way in Fig. 4, No. 1No. 6 as source electrode $(A)$ successively for data acquisition and 3-D resistivity inversion, the results are shown in Fig. 6. The 3-D resistivity inversion results can accurately reflect 
electrical property and spatial position of anomalous body. When $h=0 \mathrm{~m}$, the minimum of apparent resistivity in abnormal area located near the coal seam floor, and abnormal area has a high goodness of fit with actual position (distance of face direction and roadway direction are $40 \sim 60 \mathrm{~m}$ and $40 \sim 60 \mathrm{~m}$ respectively); when $h=20 \mathrm{~m}$, the minimum of apparent resistivity of abnormal area is located in the vicinity of $20 \mathrm{~m}$, and the abnormal area expanded, it consistent with the actual position of anomalous body along the direction of the roadway, however, the deviation increases along the working face direction; when $h=40 \mathrm{~m}$, the abnormal amplitude of 3-D resistivity inversion results is small, hence, no obvious abnormal response can be considered.
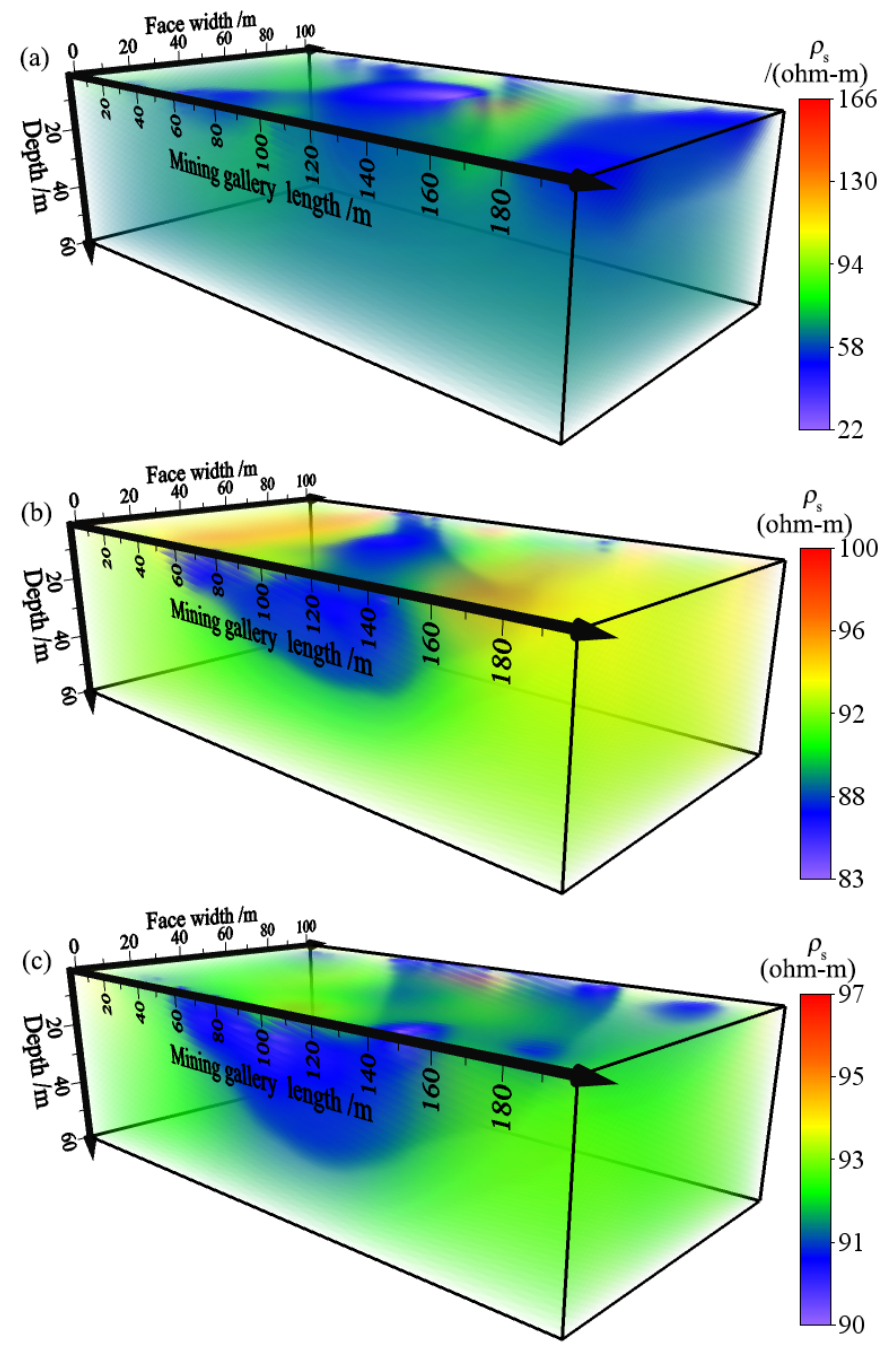

Fig.6 Stereogram of 3-D resistivity inversion of low resistance body at different depths: (a) $h=0 \mathrm{~m}$; (b) $h=20$; (c) $h=40 \mathrm{~m}$

\section{CONCLUSIONS}

Based on above researches, the following conclusions can be obtained. Firstly, response characteristics of electrical penetration curve is related to the spatial location of anomalous body, anomalous body at shallow electrical penetration curves showed opposite electrical characteristics; abnormal response is strongest when close to the measuring point and is weakest near the middle of the working face. Secondly, 3-D resistivity inversion results can accurately judge electrical property and spatial position of anomalous body, the effective exploration depth of electrical penetration is about $30 \%-40 \%$ of working face width, and increase at first then decrease from both sides toward the middle, besides, there exist exploration weakness in the middle of the working face. Lastly, concealed structure on coal seam floor can be accurately delineated by the method of data processing and interpretation of the combination based on 3-D resistivity inversion and electrical penetration curve.

\section{REFERENCES}

[1] S. D. Liu, R. X. Wu, P. S. Zhang, Y. Cao. Three dimensional parallel electric surveying and its applications in water disaster exploration in coal mines, Journal of China Coal Society (in Chinese), vol. 34, 2009, pp. 927932.

[2] S. C. Liu, J. H. Yue, and Z. D. Li. Studies on changing law of theoretical electrical sounding curves in coal mine, Journal of China University of Mining \& Technology (in Chinese), vol. 25, 1996, pp. 101-105.

[3] D. P. Han, and Y. D. Shi. The numerical simulation of the electrical penetration methods at coal mining working face in roof or floor and in coal seam, Journal of China Coal Society (in Chinese), vol. 25, 2000, pp. 30-33.

[4] Q. W. Liu, Y. L. Liu, and D. P. Han. Two dimension numerical modeling of electrical penetrative detection in coal mine, Coal Geology and Exploration (in Chinese), vol. 29, 2001, pp. 48-52.

[5] A. Gyulai, M. Dobroka, T. Ormos, E. Turai, T. Sasvari. In-mine geoelectric investigations for detecting tectonic disturbances in coal seam structures, Acta Geophysica, vol. 61, 2013, pp. 1184-1195.

[6] Y. Sasaki, M. Koichi, Surface-to-tunnel resistivity tomography at the Kamaishi mine, Butsuri Tansa, vol. 46, 1993, pp. 83-87.

[7] E. Takacs. The considering the galvanic and induction effects in the inversion of vertical electric dipole transillumination measurement, Acta Geod. Geoph. Hung, vol. 47, 2012, pp. 223-232.

[8] J. Csokas, M. Dobroka, and A. Gyulai. Geoelectric determination of quality changes and tectonic disturbances in coal deposits, Geophysical prospecting, vol. 34, 1986, pp. 1067-1081. 